\title{
ON LINKING COEFFICIENTS
}

\author{
NATHAN HABEGGER ${ }^{1}$
}

\begin{abstract}
The possible values of linking coefficients for two component links are studied. An example of a link $S^{3} \cup S^{2}$ in $S^{5}$ having linking coefficient in $\pi_{3}\left(S^{2}\right)$ of Hopf invariant two is constructed. A generalization to links $S^{m-2} \cup S^{p}$ in $S^{m}$ is obtained in the metastable range. Applications to embeddings of two cell complexes are cited.
\end{abstract}

0. Introduction and statement of results. Let $f: S^{a} \rightarrow S^{m}, g: S^{b} \rightarrow S^{m}$ be a pair of disjoint (pl) embeddings of spheres (a two component link). The complement $S^{m} \backslash g\left(S^{b}\right)$ has the homotopy type of $S^{m-b-1}$ if either $b \leqslant m-3$ or $b=m-2$ and $g$ is unknotted. (If $b \leqslant m-3$, then by Zeeman [Z] $g$ is unknotted.) In either case, $f$ represents an element of $\pi_{a}\left(S^{m-b-1}\right)$ called a linking coefficient, which we denote by $I,(f, g)$.

Problem A. What values can $L(f, g)$ take?

The following theorem restricts the possible values of $L(f, g)$ (cf. Kervaire [K]).

TheOREM 1. Suppose $L(f, g)$ and $L(g, f)$ are both defined. Then $\Sigma^{b} L(f, g)=$ $\pm \Sigma^{a} L(g, f)$ in $\pi_{a+b}\left(S^{m-1}\right)$, where $\Sigma^{b}, \Sigma^{a}$ are iterated suspension homomorphisms.

Note that $\pi_{a+b}\left(S^{m-1}\right)$ is a stable homotopy group. Hence,

Corollary 2. Suppose $m-a \geqslant 3, m-b \geqslant 3$. A necessary condition for $L \in$ $\pi_{a}\left(S^{m-b-1}\right)$ to be a linking coefficient is that L stably desuspends to $\pi_{b}\left(S^{m-a-1}\right)$.

Using Haefliger's link classification theorem $[\mathbf{H}]$ in codimension greater than two, the above condition is seen to be sufficient in a range.

TheOrem 3. Suppose $m-a \geqslant 3, m-b \geqslant 3$ and $2 a+2 b \leqslant 3 m-6$. Suppose $L$ stably desuspends to $\pi_{b}\left(S^{m-a-1}\right)$. Then $L$ is a linking coefficient for some link $S^{a} \cup S^{b}$ in $S^{m}$.

W. Massey has asked: What can be said if one of the factors is allowed to have codimension 2? For links $f: S^{m-2} \rightarrow S^{m}, g: S^{p} \rightarrow S^{m}, q=m-q-1 \geqslant 2$, we have $L(f, g)$ defined in $\pi_{m-2}\left(S^{q}\right)$. However, because of possible knotting of the

Received by the editors October 10, 1984.

1980 Mathematics Subject Classification. Primary 57Q35, 57Q45, 57R40; Secondary 54C25, 54E60, $55 \mathrm{Q} 25$.

Key words and phrases. Links, linking coefficients, Hopf invariant, cell complexes, embeddings, knots.

${ }^{1}$ Partially supported by the National Science Foundation, grant \# DMS 84-01578. 
codimension 2 factor, $L(g, f)$ need not be defined. Nevertheless, Levine (unpublished; cf. [F] for an alternative proof) has shown that Kervaire's proof can be modified to show that $L(f, g)$ is stably trivial $(p \geqslant 2)$. A stronger result is in fact true:

THEOREM 4. Let $f: S^{m-2} \rightarrow S^{m}, g: S^{p} \rightarrow S^{m}$ be disjoint embeddings $q=m-p-$ $1 \geqslant 2, p \geqslant 2$, Then the (single) suspension $\Sigma L(f, g) \in \pi_{m-1}\left(S^{q+1}\right)$ of $L(f, g)$ is zero.

This necessary condition is sufficient in the metastable range:

Theorem 5. Suppose $p \geqslant 2, q=m-p-1 \geqslant 2$ and $m-2 \leqslant 3 q-3$. Suppose $L \in \pi_{m-2}\left(S^{q}\right)$ satisfies $\Sigma L=0$, where $\Sigma L \in \pi_{m-1}\left(S^{q+1}\right)$ is the suspension of $L$. Then there is a link $S^{m-2} \cup S^{p}$ with linking coefficient L. Moreover, $S^{m-2}$ may be taken to be unknotted.

REMARK. Since $S^{m-2}$ may be taken unknotted, it follows that $S^{m-2}$ can have any knot type. This is surprising since it had been thought that knotting phenomena might give rise to "obstructions".

Links $S^{3} \cup S^{2}$ are a particular case of Theorem 5. Theorems 4 and 5 can be restated as follows:

Corollary 6. $\alpha \in \pi_{3}\left(S^{2}\right)$ is the linking coefficient of some $S^{3} \cup S^{2}$ in $S^{5}$ if and only if $\alpha$ has even Hopf invariant.

There is a close relationship between links and embeddings of two cell complexes up to homotopy type. Let $S^{n-1} \rightarrow S^{m} \backslash S^{p}$ be an embedding. Consider $S^{m} \subset D^{m+1}$ $\subset \mathbf{R}^{m+1}$. Then "coning off" the embedding yields an embedding up to homotopy type of $S^{q} \cup_{\alpha} D^{n}$ in $\mathbf{R}^{m+1}$, where $\alpha$ is the homotopy class of $S^{n-1} \rightarrow S^{m} \backslash S^{p} \simeq S^{q}$, $q=m-p-1 \geqslant 2$. Therefore, Theorem 5 yields

COROllary 7. Suppose $q \geqslant 2, n-q \geqslant 2$ and $n \leqslant 3 q-2$. Let $\alpha \in \pi_{n-1}\left(S^{q}\right)$ satisfy $\Sigma \alpha=0$. Then $S^{q} \cup_{\alpha} e^{n}$ embeds up to homotopy type in $\mathbf{R}^{n+2}$.

It seems likely that the condition $\Sigma \alpha=0$ is necessary. We can prove

THEOREM 8. If $S^{q} \cup_{\alpha} e^{n}$ embeds in $\mathbf{R}^{n+2}, n-q \geqslant 2$, then $\alpha$ is stably trivial.

Corollary 9. $S^{2} \cup_{\alpha} e^{4}$ embeds in $\mathbf{R}^{6}$ up to homotopy type if and only if $\alpha$ has even Hopf invariant.

(That $S^{2} \cup_{\alpha} e^{4}$ does not embed in $\mathbf{R}^{6}$, for $\alpha$ of odd Hopf invariant, is classical and due to Thom [T].)

This paper is organized as follows. In $\S 1$ we recall known results in codimension greater than 2. In $\$ 2$ we given an explicit embedding of $S^{3} \cup S^{2}$ in $S^{5}$ with linking coefficient of Hopf invariant 2. The more general result, Theorem 5, is proved in $\S 3$. We make use of explicit embeddings of Hacon [Hac] and calculate their linking coefficients. Finally, proofs of Theorems 4 and 8 are given in $\S 4$. They both have the same homotopy theoretical flavor.

The interested reader may refer to [Ke] for a generalization of Hacon's results. 
1. Haefliger's classification sequence. The following result is due to Zeeman (cf. $[\mathbf{Z}])$.

Proposition. Suppose $a \leqslant b$. Then any $L \in \pi_{a}\left(S^{m-b-1}\right)$ is the linking coefficient of some link.

Proof. If $g: S^{b} \rightarrow S^{m}$ is the standard embedding, then $S^{m} \backslash g\left(S^{b}\right)=S^{m-b-1} \times$ $\mathbf{R}^{b+1}$. Let $\varphi: S^{q} \rightarrow S^{m-b-1}$ represent $L$, and let $\psi: S^{a} \rightarrow \mathbf{R}^{b+1}$ be the standard embedding $S^{a} \subset \mathbf{R}^{a+1} \subset \mathbf{R}^{b+1}$ (or any embedding). Then $f=(\varphi, \psi): S^{a} \rightarrow S^{m-b-1}$ $\times \mathbf{R}^{b+1}$ is also an embedding, and $L(f, g)=L$.

In $[\mathbf{H}]$, Haefliger reduces the classification problem for links of codimension greater than 2 to a problem in homotopy theory. As a particular case, he proves

TheOREM (HAefliger [H]). Suppose $m-a \geqslant 3, m-b \geqslant 3, b \leqslant a$ and $b+3 a \leqslant$ $3 m-6$. Let $L_{a, b}^{m}$ denote the group of isotopy classes of pl links $S^{a} \cup S^{b}$ in $S^{m}$. There is an exact sequence

$$
L_{a, b}^{m} \stackrel{\lambda}{\rightarrow} \pi_{a}\left(S^{m-b-1}\right) \oplus \pi_{b}\left(S^{m-a-1}\right) \stackrel{\omega}{\rightarrow} \pi_{m-2}\left(S^{2 m-a-b-3}\right),
$$

where $\lambda(f, g)=(L(f, g), L(g, f))$ and $\omega$ is the iterated suspension (up to sign) on each factor.

REMARKS. (1) W. Massey has pointed out that the hypothesis $b+3 a \leqslant 3 m-6$ may be replaced by the weaker hypothesis $2 b+2 a \leqslant 3 m-6$. This is just the hypothesis necessary so that the group $\pi_{m-2}\left(S^{2 m-a-b-3}\right)$ is stable. The proof is as in $[\mathbf{H}]$.

(2) Theorem 3 is an immediate consequence of the exactness of this sequence and the above remark.

(3) The homomorphism $\omega$ expresses a symmetry in the linking coefficients, which we recall here for use later.

Let $M$ be the boundary connected sum of $S^{a} \times D^{m-a}$ and $S^{b} \times D^{m-b}$. Given an embedding of $S^{a} \cup S^{b}$ in $S^{m}$, by Zeeman (since each component is unknotted) we may extend the embeddings to framed embeddings and hence to an embedding of $M$ (unique up to concordance).

Let $a^{\prime}=m-a-1, b^{\prime}=m-b-1$. Then $\partial M=S^{a} \times S^{a^{\prime}} \# S^{b} \times S^{b^{\prime}}$. One can easily check that the inclusion $S^{a \prime} \vee S^{b \prime} \rightarrow \overline{S^{m} \backslash M}$ is a homotopy equivalence. Restricting a homotopy inverse to $\partial M$ yields a retraction $\lambda: S^{a} \times S^{a \prime} \# S^{b} \times S^{b^{\prime}} \rightarrow$ $S^{a \prime} \vee S^{b \prime}$.

Let $\lambda_{a}, \lambda_{b}$ be the homotopy classes of the maps $\left.\lambda\right|_{S^{a}},\left.\lambda\right|_{S^{b}}$. These are generalized linking invariants in $\pi_{*}\left(S^{a \prime} \vee S^{b \prime}\right)$. Let $i_{a}$ denote the homotopy class of the inclusion $S^{a \prime} \rightarrow S^{a \prime} \vee S^{b \prime}$, and let $p_{a}$ denote the homotopy class of the projection $S^{a \prime} \vee S^{b \prime} \rightarrow S^{a \prime} ;$ similarly for $i_{b}, p_{b}$. Then $\lambda_{a}, \lambda_{b}$ satisfy

(1) $p_{a} \circ \lambda_{a}=0, p_{b} \circ \lambda_{b}=0$,

(2) $p_{b} \circ \lambda_{a}=L(f, g), p_{a} \circ \lambda_{b}=L(g, f)$,

(3) $\left[\lambda_{a}, i_{a}\right]+\left[\lambda_{b}, i_{b}\right]=0$, where $[$, ] denotes Whitehead product.

Condition (3) is the desired symmetry relation. 
2. An example of a link of $S^{3}$ and $S^{2}$ in $S^{5}$. Consider $S^{2} \times \mathbf{R}^{3}=S^{5} \backslash S^{2} \subset S^{5}$. Let $f: S^{2} \vee S^{2} \rightarrow S^{2} \times \mathbf{R}^{3}$ be any embedding which up to homotopy is just the folding map $\Lambda: S^{2} \vee S^{2} \rightarrow S^{2}$. Suppose $f$ extends in $S^{5}$ to an embedding $S^{2} \times S^{2}$ $\rightarrow S^{5}$. Some small neighborhood $X$ of $S^{2} \vee S^{2}$ in $S^{2} \times S^{2}$ will be embedded into $S^{2} \times \mathbf{R}^{3}$. The resulting embedding $S^{3}=\partial X \rightarrow X \rightarrow S^{2} \times R^{3}=S^{5} \backslash S^{2}$ will have Hopf invariant two (since $\partial X \rightarrow X$ is, up to homotopy, the Whitehead product $\left[i_{1}, i_{2}\right]: S^{3} \rightarrow S^{2} \vee S^{2}$ and $\Lambda \circ\left[i_{1}, i_{2}\right]=[i, i]$ has Hopf invariant two). Moreover, since $\partial X$ bounds a disk in $S^{2} \times S^{2}$, this embedding is unknotted in $S^{5}$.

It is an easy matter to embed $S^{2} \vee S^{2}$ as above: Let $D_{1}^{3} \subset \mathbf{R}^{3}$ be a disk and $S_{1}^{2}=\partial D_{1}^{3}$. The embedding $S^{2} \vee S_{1}^{2} \subset S^{2} \times S_{1}^{2} \subset S^{2} \times \mathbf{R}^{3}$ satisfies all the conditions above except that $S_{1}^{2} \rightarrow S^{2} \times \mathbf{R}^{3}$ is of degree zero. Choose $* \in \mathbf{R}^{3}$ outside of $D_{1}^{3}$. Define a new embedding $S^{2} \vee S_{1}^{2} \# S^{2} \times *$ (see the diagram), where \# denotes the connected sum along some path joining the two spheres. This now has degree 1 on the second factor. Moreover, since $S^{2} \times *$ bounds a disk in $S^{5} \backslash S^{2} \times D_{1}^{3}$, the two embeddings are ambient isotopic in $S^{5}$. In particular, the new embedding also extends to $S^{2} \times S^{2}$, as required.

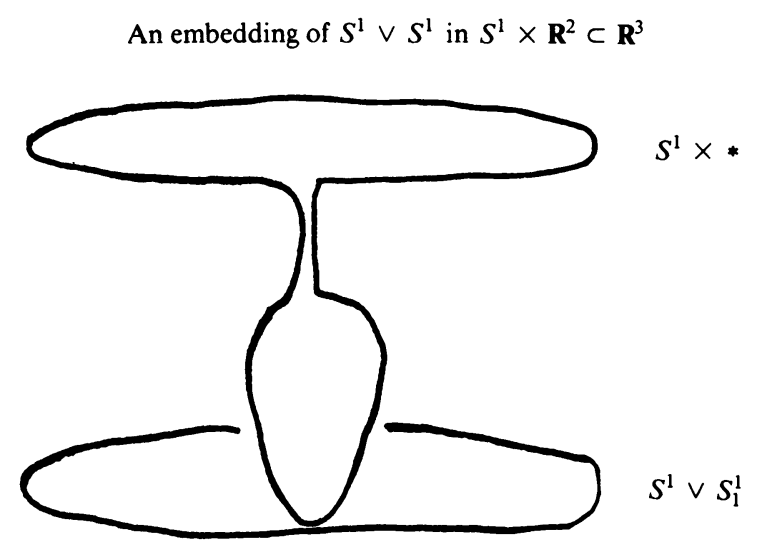

REMARK. The link $S^{3} \cup S^{2} \subset S^{5}$ may also be obtained as follows: Note that since $f\left(S^{3}\right) \subset S^{5}$ is unknotted, $S^{5} \backslash f\left(S^{3}\right)=S^{1} \times \mathbf{R}^{4}$. Then $g: S^{2} \rightarrow S^{1} \times \mathbf{R}^{4}$ is an embedding which, while homotopically trivial, cannot be isotopic to the trivial embedding.

In [Hac], Hacon shows that embeddings $S^{2} \hookrightarrow S^{1} \times \mathbf{R}^{4}$ are classified up to isotopy by the following: Let $\overline{S^{1} \times \mathbf{R}^{4}}=\mathbf{R}^{5}$ be the universal cover with transformation group $\langle T\rangle=\mathbf{Z}$. Then if $\tilde{g}: S^{2} \rightarrow S^{1} \times \mathbf{R}^{4}$ is any lift of $g: S^{2} \rightarrow S^{1} \times \mathbf{R}^{4}$, the linking numbers $L\left(\tilde{g}, T^{k} \tilde{g}\right) \in \mathbf{Z}, k \neq 0$, are defined and nonzero for only finitely many $k$. The map $\operatorname{Emb}\left(S^{2}, S^{1} \times \mathbf{R}^{4}\right) \rightarrow \bigoplus_{k=1}^{\infty} \mathbf{Z}$ is a bijection.

Follow the isotopy in the construction of the link backwards. One gets an isotopy of $g\left(S^{2}\right)$ (where $S^{5} \backslash g\left(S^{2}\right)=S^{2} \times \mathbf{R}^{3}$ ) to a new embedding $g^{\prime}: S^{2} \rightarrow S^{5}$ in the complement of $S^{2} \vee S_{1}^{2}$ linking each wedge factor once. Up to isotopy (since any two such are isotopic) it is precisely the connected sum of $g\left(S^{2}\right)$ and $S^{2} \times 0 \subset S^{2}$ $\times D^{3} \subset S^{2} \times \mathbf{R}^{3}$. Note that $g\left(S^{2}\right)$ and $S^{2} \times 0$ link once. Since the path connecting 
them travels once through $D^{3}=S^{2} \times S^{2} \backslash S^{2} \vee S^{2}$, we get

$$
L\left(\tilde{g}^{\prime}, T^{k} \tilde{g}^{\prime}\right)= \begin{cases}1, & k=1 \\ 0, & k>1\end{cases}
$$

3. Proof of Theorem 5. By Hacon [Hac], embeddings of $S^{p}$ in $S^{1} \times R^{m-1}=$ $S^{m} \backslash S^{m-2}$ form a group isomorphic to $\oplus_{k=1}^{\infty} \pi_{p}\left(S^{q}\right), q=m-p-1$, valid in the metastable range $3(p+1)<2 m$; i.e., $m-2 \leqslant 3 q-3$. If $\langle T\rangle=\pi_{1}\left(S^{1} \times \mathbf{R}^{m-1}\right)$ denotes the deck transformation group for the universal covering $\mathbf{R}^{m}=S^{1} \times \mathbf{R}^{m-1}$, the Hacon invariants are the linking coefficients $L\left(\tilde{g}, T^{k} \tilde{g}\right) \in \pi_{p}\left(S^{q}\right)$, where $\tilde{g}$ : $S^{p} \rightarrow R^{m}=S^{1} \times R^{m-1}$ denotes any lift of $g: S^{p} \rightarrow S^{1 \times \mathbf{R}^{m}-1}$. Note that this is independent of the choice of lifts.

Associate to any embedding $g: S^{p} \rightarrow S^{1} \times \mathbf{R}^{m-1}=S^{m} \backslash S^{m-2}$ a link $(f, g)$ : $S^{m-2} \cup S^{p} \subset S^{m}$. One obtains from this link the linking coefficient $L(f, g)$ in $\pi_{m-2}\left(S^{m-p-1}\right)$.

Using Hacon's isomorphism, this gives a map $\oplus_{k=1}^{\infty} \pi_{p}\left(S^{q}\right) \rightarrow \pi_{m-2}\left(S^{q}\right)$ which can be calculated:

Proposition. The above map is given by the formula

$$
\bigoplus \beta_{k} \rightarrow[i, i] \circ \sum_{k=1}^{\infty} k \tilde{\beta}_{k},
$$

where $\tilde{\beta}_{k} \in \pi_{m-2}\left(S^{2 q-1}\right)$ is (up to sign) the iterated suspension of $\beta_{k}$.

To prove Theorem 5 from the Proposition, note that under the hypothesis of the theorem, $\pi_{p}\left(S^{q}\right)$ is a stable group. Taking $\beta_{1}=\beta$ and $\beta_{k}=0, k \neq 1$, we see that the right side is of the form $[i, i] \circ \tilde{\beta}$, with $\tilde{\beta}$ any arbitrary element of $\pi_{m-2}\left(S^{2 m-2 p-3}\right)$. Furthermore, under the hypothesis $m-2 \leqslant 3 q-3$, the EHP sequence [W, pp. 548-549] is valid. It follows that any element in the kernel of the suspension homomorphism is of the form $[i, i] \circ \tilde{\beta}$. Thus if $L$ is in the kernel, let $L=[i, i] \circ \tilde{\beta}$. Then $L$ is the linking coefficient of the link $S^{m-2} \cup S^{p}$ having Hacon invariant $\beta_{1}=\beta, \beta_{k}=0, k>1$.

Proof of the Proposition. As in $\$ 1$, Remark (3), extend the embedding $S^{m-2} \cup S^{p} \subset S^{m}$ to an embedding of the compact manifold $M$, boundary connected sum of $S^{m-2} \times D^{2}$ and $S^{p} \times D^{q+1}$. $\partial M=S^{m-2} \times S^{1} \# S^{p} \times S^{q}$, and the inclusion $S^{1} \vee S^{q} \rightarrow \overline{S^{m} \backslash M}$ is again a homotopy equivalence. The restriction of a homotopy inverse to $\partial M$ yields a retraction $\lambda: S^{m-2} \times S^{1} \# S^{p} \times S^{q} \rightarrow S^{1} \vee S^{q}$.

The Hacon invariants are contained in the homotopy class of the map (denoted by B) $\left.\lambda\right|_{S^{n}}: S^{p} \rightarrow S^{1} \vee S^{q}$. The desired linking coefficient $L(f, g)$ is obtained from the homotopy class (denoted by $\alpha$ ) of the map $\left.\lambda\right|_{S^{m-2}}: S^{m-2} \rightarrow S^{1} \vee S^{q}$ by composing with the projection to $S^{q}$. Furthermore, $\alpha$ and $\beta$ are related by the formula

$$
(T-1) \alpha+[\beta, i]=0,
$$

where $i$ is the homotopy class of the inclusion $S^{q} \rightarrow S^{1} \vee S^{q}$, and $\langle T\rangle=$ $\pi_{1}\left(S^{1} \vee S^{q}\right)$ acts on $\pi_{*}\left(S^{1} \vee S^{q}\right)$ in the usual fashion. Now since $T-1$ is injective, we are able to calculate $\alpha$ from $\beta$. 
Under the dimension restrictions, $m-2 \leqslant 3 q-3$ (i.e., $p<2 q-1$ ), $\pi_{p}\left(S^{1} \vee S^{q}\right)=\oplus_{k=-\infty}^{\infty} \pi_{p}\left(S^{q}\right)$, and so we may write $\beta=\sum_{k=-\infty}^{\infty} T^{k}\left(i \circ \beta_{k}\right)$ (this is a finite sum). For $k \neq 0, \beta_{k}$ is just the linking coefficient $L\left(\tilde{g}, T^{k} \tilde{g}\right) \in \pi_{p}\left(S^{q}\right)$.

From Remark (3) of $\S 1$, the symmetry of the linking coefficients $L\left(\tilde{g}, T^{k} \tilde{g}\right)$ and $L\left(T^{k} \tilde{g}, \tilde{g}\right)=L\left(\tilde{g}, T^{-k} \tilde{g}\right)$ can be written as

$$
\left[T^{k}\left(i \circ \beta_{k}\right), i\right]+\left[i \circ \beta_{-k}, T^{k} i\right]=0 ;
$$

i.e.,

$$
\left[T^{-k}\left(i \circ \beta_{-k}\right), i\right]=-T^{-k}\left[T^{k}\left(i \circ \beta_{k}\right), i\right]
$$

Hence

$$
(T-1) \alpha=[\beta, i]=\left[i \circ \beta_{0}, i\right]+\sum_{k=1}^{\infty}\left(1-T^{-k}\right)\left[T^{k}\left(i \circ \beta_{k}\right), i\right] .
$$

Put $T=1$ in the above formula. We obtain $\left[i \circ \beta_{0}, i\right]=0$. Hence, since $T-1$ is injective, we get that

$$
\alpha=\sum_{k=1}^{\infty} T^{-k}\left(1+T+\cdots+T^{k-1}\right)\left[T^{k}\left(i \circ \beta_{k}\right), i\right] .
$$

Putting $T=1$ gives

$$
L(f, g)=\sum_{k=1}^{\infty} k\left[\beta_{k} \circ i, i\right] .
$$

Since $\beta_{k}$ is stable (cf. [W]), this is equal to $[i, i] \circ \sum_{k=1}^{\infty} k \tilde{\beta}_{k}$, where $\tilde{\beta}_{k}$ is (up to sign) the iterated suspension of $\beta_{k}$.

\section{Proofs of Theorems 4 and 8.}

Proof of Theorem 8. Let $X=S^{n+2} \backslash S^{k} \cup_{\alpha} e^{n}$. Then $X$ is stably homotopy equivalent to $S^{k} \cup_{\alpha} e^{n}$ (cf. [C or Ha]). Now $H^{1}(X)=H_{n}\left(S^{k} \cup_{\alpha} e^{n}\right)=\mathbf{Z}$. Thus there is a map $X \rightarrow S^{1}$ inducing an isomorphism on $H_{1}$. Hence stably, $X$ retracts onto the bottom cell, and hence $\alpha$ is stably trivial.

Proof of Theorem 4. Put $K=\overline{S^{m} \backslash N\left(S^{m-2}\right)}, \quad K_{X}=\overline{S^{m} \backslash N(X)}, \quad X=S^{p}$, where $N\left(S^{m-2}\right), N(X)$ are regular neighborhoods. Consider the diagram

$$
\begin{array}{ccc}
S^{1} \times S^{m-2} & \stackrel{1 \times L}{\rightarrow} & S^{1} \times K_{X} \\
\downarrow & \searrow \rho & \downarrow \\
S^{1} \wedge S^{m-2} & \stackrel{\Sigma L}{\rightarrow} & S^{1} \wedge K_{X}
\end{array}
$$

The map $\rho$ sends $S^{1} \vee S^{m-2}$ to the basepoint. We must show that $\rho$ is homotopic to a constant map rel $S^{1} \vee S^{m-2}$.

In fact, it suffices to show that $\rho$ is homotopic to a constant by a homotopy only rel $S^{1}$ and not rel $S^{1} \vee S^{m-2}$. For if $h_{t}$ is a homotopy (rel $S^{1}$ ) of $\rho$ to a constant, then $g_{t}=\left.h_{1-t}\right|_{S^{m-2}} \circ p_{2}$ (where $p_{2}: S^{1} \times S^{m-2} \rightarrow S^{m-2}$ is the projection) is a self-homotopy $\left(\operatorname{rel} S^{1}\right)$ of the constant map $S^{1} \times S^{m-2} \rightarrow S^{1} \wedge K_{X}$, which on $S^{m-2}$ 
is inverse to $h_{t}$. The combined homotopy

$$
H_{t}= \begin{cases}h_{2 t}, & t \leqslant 1 / 2, \\ g_{2 t-1}, & t \geqslant 1 / 2,\end{cases}
$$

may therefore be homotoped to a homotopy (rel $S^{1} \vee S^{m-2}$ ) of $\rho$ to a constant.

Now $N\left(S^{m-2}\right)=D^{2} \times S^{m-2}$ (we are assuming $S^{m-2} \rightarrow S^{m}$ is locally flat). Hence $\partial K=\partial N\left(S^{m-2}\right)=S^{1} \times S^{m-2}$. Suppose we show that $\rho$ can be extended to a map $K \rightarrow S^{1} \wedge K_{X}$. Then since $S^{1} \rightarrow K$ is a homology equivalence, and $S^{1} \rightarrow S^{1}$ $\wedge K_{X}$ is the constant map, it follows that $K \rightarrow S^{1} \wedge K_{X}$ (and hence $S^{1} \times S^{m-2} \rightarrow$ $\left.S^{1} \wedge K_{X}\right)$ is homotopic $\left(\operatorname{rel} S^{1}\right)$ to a constant map.

Thus it suffices to extend $\rho$ to a map $K \rightarrow S^{1} \wedge K_{X}$. To do this, note that, since $H^{1}(K)=H^{1}\left(S^{1} \times S^{m-2}\right)=H^{1}\left(S^{1}\right)=\mathbf{Z}$ and since $S^{1}$ is a $K(\mathbf{Z}, 1)$, the projection $S^{1} \times S^{m-2} \rightarrow S^{1}$ may be extended to a map $\varphi: K \rightarrow S^{1}$. Moreover, since (by hypothesis) $H_{1}(X) \rightarrow H_{1}(K)$ is trivial, we may assume that $\varphi$ sends $N(X)$ to the basepoint.

Now the map $S^{1} \times S^{m-2} \rightarrow S^{m-2} \subset K_{X}$ extends to $K \cap K_{X} \rightarrow K_{X}$ (which, up to homotopy, is just the inclusion). Thus the map $S^{1} \times S^{m-2} \stackrel{1 \times L}{\rightarrow} S^{1} \times K_{X}$ extends to a map $K \cap K_{X} \rightarrow S^{1} \times K_{X}$ such that the composite $K \cap K_{X} \rightarrow S^{1} \wedge K_{X}$ sends $\partial N(X)$ to the basepoint. We may thus extend $\rho$ to all of $K$ by sending $N(X)$ to the basepoint.

ACKNOWLEDGEMENTS. Theorem 4 is a homotopy theoretical version of a geometric proof for $X$ a sphere and $S^{m-2}$ unknotted which grew out of a discussion $I$ had with Tim Cochran and Jerry Levine. I wish to thank W. Massey for stimulating my interest in these questions and for the many hours of collaboration he spent with me on this project.

\section{REFERENCES}

[C] George E. Cooke, Embedding certain complexes up to homotopy type in Euclidean space, Ann. of Math. (2) 90 (1969), 144-156.

[F] Roger Fenn, Some generalizations of the Borsuk-Ulam theorem and applications to realizing homotopy classes by embedded spheres, Proc. Cambridge Philos. Soc. 74 (1973), 251-256.

[H] A. Haefliger, Enlacements de sphères en codimension supérieure à 2, Comment. Math. Helv. 4 (1966-67), 51-72.

[Ha] N. Habegger, On the existence and classification of homotopy embeddings of a complex into a manifold, Thesis, Univ. of Geneva, 1981.

[Hac] D. Hacon, Embeddings of $S^{p}$ in $S^{1} \times S^{q}$ in the metastable range, Topology 7 (1968), 1-10.

[K] M. Kervaire, An interpretation of $G$. Whitehead's generalization of $H$. Hopf's invariant, Ann. of Math. (2) 69 (1959), 345-365.

[Ke] C. Kearton, Obstructions to embedding and isotopy in the metastable range, Math. Ann. 243 (1979), $103-113$.

[T] R. Thom, Espaces fibrés en sphères et carrés de Steenrod, Ann. Sci. École Norm. Sup. (4) 69 (1952), $109-182$.

[W] G. Whitehead, Elements of homotopy theory, Grad. Texts in Math., Springer-Verlag, Berlin and New York.

[Z] E. C. Zeeman, Unknotting combinatorial balls, Ann. of Math. (2) 78 (1963), 501-526.

Department of Mathematics, Yale University, New Haven, Connecticut 06520

Current address: Department of Mathematics, University of California at San Diego, La Jolla, California 92093 